\title{
Material and shape selection for stiff beams under non-uniform flexure
}

\author{
A. Amany and D. Pasini ${ }^{1}$ \\ Mechanical Engineering Department, McGill University \\ ${ }^{1}$ Corresponding author: damiano.pasini@mcgill.ca \\ Mechanical Engineering Department, McGill University \\ Macdonald Engineering Building, room 372 \\ 817 Sherbrooke Street West \\ Montreal, Quebec, H3A2K6, Canada \\ tel. (1) 5143986295 \\ fax. (1) 5143987365
}

A shape and material selection model has been previously introduced to characterize the structural efficiency of a slender beam under pure bending. The method is extended here and applied to the case of a beam undergoing non-uniform flexure. In the first part of this paper, the strain energy density is used to formulate the beam stiffness in terms of strain energies due to both transverse shear forces and bending moments. Shape transformers are defined to capture the shear deformation that shapes of alternative crosssection exhibit with respect to beam slenderness. In the second part, the expressions of the shape properties are plotted on to selection charts that assist co-selecting the best material, shape and slenderness of a lightweight stiff beam under non-uniform bending. Numeric results from finite element analysis validate the model for concept design.

Keywords: performance indices $(\mathrm{H})$; material selection charts $(\mathrm{H})$; selection for material properties (H).

\section{Introduction}

Optimized slender and short-thick components are used in building, aircraft and machine structures to increase performance and lower material cost. Whereas the former exist in most applications, the latter are frequently encountered in concrete and steel structures. Squat beams, for example, contribute to stiffen foundation walls and increase the load capacity of walls supported by columns [1]. Short beams with thin webs are also an integral part of aircraft structures, and short-thick cantilevers are used for gear teeth $[2,3]$. 
The strain response of a beam strongly depends on the type of applied load. In practice, most beams are subjected to loads that induce non-uniform bending, which consists of both bending moments and shear forces. Once deformed, the beam stores strain energy due to normal and shear stresses. In a slender beam, however, the strain energy of shear is negligible since it is relatively small in comparison with the strain energy due to bending. Typically, for a length-to-height ratio as high as 10 the shear deformation is less than $3 \%$ and the beam can be assumed to undergo pure flexure. On the other hand, in a short-thick beam, the pure bending assumption ceases to hold. The shear strain cannot be ignored, because a beam slenderness as large as 0.2 induces a shear deflection which is at least $10 \%$ of that caused by bending [4].

Besides slenderness and applied load, structural geometry and material are factors governing deformation and mass-efficiency. Several researchers have developed design methods to optimize the material and geometry selection. Most of them, e.g. Shanley [5], Cox [6], Parkhouse [7], Ashby[8], and Pasini $[9,10]$, introduced shape parameters, performance criteria, and selection charts to visualize the impact of design variables on structural efficiency. Cox and Shanley, for example, used a structure-loadingcoefficient for a visual comparison of the mass-efficiency of structural concepts. Ashby $[8,11]$ proposed a material index to characterize the efficiency of a material, and introduced the well-known material-property charts, an invaluable tool for material selection. In his work, Ashby [8] assumed that the impacts of material and shape are not independent and, subsequently, introduced a shape factor in the material index to allow for material and shape co-selection. More recently, a selection model has been presented to compare the lightweight potential of shaped materials on shape property charts. Based on a shape classification, the method introduces shape transformers to characterize the role of shape and decouple it from the size effect $[9,10,12,13]$.

In the design for stiffness, the aforementioned methods may be applied to non-uniform bending, as long as the beam is slender $[4,14,15]$. Their inaccuracy, however, amplifies with the increase of the beam slenderness ratio. This paper examines this last issue by focusing on the effect that shear strain and slenderness have on the co-selection of material and geometry. A method, previously introduced for pure bending [10], is extended to include non-uniform flexure and is reformulated for the lightweight design of beams of any slenderness. In section 2 , the stiffness of a beam is modeled for compliance due to combined shear and bending loading. Shape transformers are introduced in sections 3, 4 and 6 to model beam 
stiffness and performance of both slender and short-thick beams. The impact of slenderness is demonstrated in section 5 followed by design maps in section 7 . Section 8 presents finite element analysis results to validate the model.

\section{Beam Stiffness for non-uniform Bending}

Under non-uniform bending, the beam resistance to deformation can be expressed as a function of the shear and bending stiffness. In this work, the beam cross-section is assumed to be uniform along its length with homogeneous and isotropic material. The longitudinal neutral axis is linear and coincides with the $\mathrm{x}$ axis, and the orientations of $y$ - and z-axes are respectively along the height and width of the cross-section.

Consider a centrally loaded and simply supported beam undergoing small and linear-elastic deformation. The beam is modelled as a system of two springs mounted in series under a load P, similarly to the model of a beam under non-uniform bending in [16]. One spring is associated to the bending

deflection, $\delta_{b}$, at the location of the applied load. The other governs the shear deflection, $\delta_{s}$, at the same location. Therefore, the beam stiffness, $k$, can be expressed as a function of the individual stiffness due to bending, $k_{b}=P / \delta_{b}$, and shear, $k_{s}=P / \delta_{s}$, as:

$$
k=\left(\frac{1}{k_{b}}+\frac{1}{k_{s}}\right)^{-1}=\frac{P}{\delta_{b}+\delta_{s}}
$$

In the above expression, the elastic deflection $\delta=\delta_{b}+\delta_{s}$ is unknown. To compute the two spring deflections, we apply the Castigliano's theorem to the total strain energy density of the beam subjected to bending and shear forces $[2,4,17]$. In the case of non-uniform bending, the total strain energy due to a state of bending and shear stresses, $\sigma$ and $\tau$, can be expressed as:

$$
U=\int_{V}\left(u_{b}+u_{s}\right) d V=\int_{V}\left(\frac{\sigma^{2}}{2 E}+\frac{\tau^{2}}{2 G}\right) d V
$$


where $u_{b}$ and $u_{s}$ are the bending and shear strain energy per unit volume; $E$ and $G$ are respectively the Young's and shear modulus. The next step in calculating the beam deflection is to apply Castigliano's theorem at the location of the applied load. By differentiating the integral of the strain energy with respect to the load applied at the location of deformation and by substituting the internal bending and shear stresses with the external force, we obtain:

$$
\delta=\left|\delta_{b}+\delta_{s}\right|=\frac{\partial U}{\partial P}=\frac{P L^{3}}{\beta E I}+\frac{P L K}{\alpha G A}
$$

which, substituted in (1), gives the following beam stiffness:

$$
k=\left(\frac{L^{3}}{\beta E I}+\frac{L K}{\alpha G A}\right)^{-1}
$$

where $\alpha$ and $\beta$ are constants dependent on boundary conditions and load location. For a simply supported beam, the constants are found to be $\beta=48$ and $\alpha=4$ [2]; furthermore, $K$ is the shear correction factor dependent on the shear stress distribution, which in a solid rectangular cross-section is parabolic with respect to the height.

The shear correction factor is governed by the cross-section shape. Approximate expressions of $\mathrm{K}$ are available in the literature for basic cross-sections [4,18-20]. For example, in Timoshenko beam theory, a shear correction factor is introduced to compensate for the error due to assuming a constant shear stress in the cross-section. The following describes a method to model the shape properties of a cross-section and it will be used in section 4.1 to formulate $K$ for alternative shapes.

\section{Methodology}


The scheme used in this paper is based on the notion that the geometry of a cross-section can be described by two distinct entities [10]. One governs the size of the cross-section, the other its shape. The former is represented, in two-dimensions, by a rectangle, namely the envelope, $D$, of dimensions ( $B, H$ ) equal to those of the cross-section. The latter is the shape, $S$, of the figure enclosed in $D$ with geometric properties described by dimensionless parameters called shape transformers. Decoupling $D$ from $S$ leads to the definition of scalar operators that deal with the scaling and the shaping of a cross-section. The former is described by envelope multipliers; the latter by shape transformers, which represent the geometric properties of a shape, similarly to the physical properties of a material.

A shape transformer $\psi_{g}$ is defined by normalizing a geometric characteristic, $g$, of the cross-section shape by that of the envelope as:

$$
\psi_{g}=g / g_{D}
$$

where $g$ represents a geometric quantity of the shape, such as the area, $A$, or the second moment of area, $I$, and $g_{D}$ that of the envelope. Shape transformers are generally expressed in terms of $c=b / B$ and $d=h / H$, with $0 \leq c \leq 1$ and $0 \leq d \leq 1$. When the material saturates the envelope completely, the shape is a solid rectangle, which is chosen to be a reference shape with $\psi_{g}$ unity; on the other hand, $0<\psi_{g}<1$ for all other shapes.

The above scheme has been used to categorize shape concepts. Shapes have been classified into families and classes in a way similar to material classification [13]. Shapes falling into a family can be solid and hollow. The former should originate from the solid shape of the family. The latter falls into a family if the contour of the removed material, i.e. the internal hole, is the same as that of the external profile. Table 1 presents three shape families together with the shape classes of the rectangles, the ellipses, and the diamonds. Here, a shape class is defined by the direction in which the material layers are scaled with respect to the envelope. Shape classes are ideal limiting-cases that define the boundaries of all possible beam stiffness behavior for a given loading. They are theoretical, but represent approximately the cases of a 
bi-material system in which the low density core layers can be neglected. Three shape classes are considered in this work, namely vertical, horizontal and proportional scaled layers classes, as illustrated in Table 1.

Shape transformers generally enable to decouple the effects of various parameters in an expression of classical mechanics, such as a failure mechanism. In general, an equation of mechanics E.M., can be expressed in terms of design specifications, material, and geometric properties as

$$
\text { E.M. }=F \times M \times \psi_{g} \times g_{D}
$$

where $F$ identifies the design specifications and $M$ corresponds to material properties. $\psi_{g}$ symbolizes the shape transformers of the cross-section for a given geometric quantity $g$ of the shape, and $g_{D}$ represents the geometric quantity of the envelope.

\section{Shear Shape Transformers for Modeling Non-Uniform Bending Stiffness}

To apply the above scheme to non-uniform bending stiffness design, we need to formulate the shape transformers for pure shear stiffness. Equation (4) shows that the geometric contribution governing shear stiffness is $K / A$. In section 4.1, symbolic expressions of the shear correction factor are derived for the shape families. We use the results in section 4.2 to formulate the shape transformers and substitute them into the beam stiffness.

\subsection{The Shear Correction Factor for Shape Families}

As previously described, the shear correction factor is obtained by applying the strain energy method and Castigliano's theorem to the shape families and their classes. For hollow shapes, the first moment of inertia is evaluated separately in the first cross-section segment, $0 \leq y \leq h / 2$ or $-h / 2 \leq y \leq 0$ and in the second one, $h / 2 \leq y \leq H / 2$ or $-H / 2 \leq y \leq-h / 2,[4,21]$. 
Rectangles. For the family of the rectangles shown in Table 1, the expression of the shear correction factor $K$ is given by:

$$
K=\frac{3 \cdot\left(7 c d^{5}+8 c^{2} d^{5}-30 c d^{3}+15 c d-8 c+8\right)}{20 \cdot(1-c)(1-c d)^{-1}\left(1-c d^{3}\right)^{2}}
$$

For the reference cross-section, i.e. solid rectangle where $c=0$ and $d=0$, and for the class of horizontally scaled layers where $d=1$, the value of $K i s 6 / 5$, which is in agreement with that found in literature $[2,3,17,22]$.

Applying the scaling conditions $c=d$ and $c=1$ to Eq.(7), gives the shear correction factor respectively for the proportionally and vertically scaled layers classes. These expressions will be used in section 4.2 to formulate the shape transformers, as illustrated in Table 2.

Ellipses. Similarly, we apply the strain energy method and Castigliano's theorem to each shape class of the family of the ellipses. For the proportionally scaled hollow ellipse, the shear correction factor is given by:

$$
K=\frac{2\left(3 \pi d^{4}+8 d^{3} \sqrt{1-d^{2}}+13 \pi d^{2}+4 d \sqrt{1-d^{2}}\right)}{9 \pi\left(d^{4}+2 d^{2}+1\right)}+\frac{2\left(16 d^{2} \arcsin (d)-4 \arcsin (d)+5 \pi\right)}{9 \pi\left(d^{4}+2 d^{2}+1\right)}
$$

For a solid ellipse and for the horizontally scaled layers ellipse, the shear correction factor is correctly found to be equivalent to that of a circle [2], which is $K=10 / 9$. The shear correction factor for the vertically scaled ellipse is obtained by numeric analysis.

Diamonds. For the proportionally scaled diamond, $\mathrm{K}$ is given by: 


$$
K=\frac{8 d^{5}+56 d^{4}+101 d^{3}+61 d^{2}+31 d+31}{30\left(d^{3}+d^{2}+d+1\right)\left(1+d^{2}\right)}
$$

The shear correction factor for a solid diamond and for the horizontally scaled diamond is $K=31 / 30$, which is in agreement with [2]. The above expressions given for alternative shapes are used in the following section to formulate the shape transformers for pure shear stiffness.

\subsection{Beam stiffness for non-uniform flexure}

The shear shape transformer $\psi_{S}$ governs the pure shear correction factor of a cross-section. As defined in Eq. (5), $\psi_{S}$ is a measure of the geometric term, $A / K$, relative to that of the envelope, given by:

$$
\psi_{S} \equiv\left(A / A_{D}\right) /\left(K / K_{D}\right)
$$

Table 2 summarizes the shape transformers for the shapes illustrated in Table 1. Similar to materials, shapes of a family exhibit properties that fall into a particular range of properties. For the hollow rectangles, ellipses and diamonds, $\psi_{S}$ varies respectively as follows: $0 \leq \psi_{S} \leq 1,0 \leq \psi_{S} \leq 27 \pi / 100$ and $0 \leq \psi_{S} \leq 18 / 31$

If $\psi_{S}$ is substituted in Eq.(4), the terms of shape, material and size are decoupled in the shear stiffness, which can be expressed as:

$$
k_{S}=(\alpha / L) \cdot G \cdot \psi_{S} \cdot\left(A_{D} / K_{D}\right)
$$

In addition to material and geometry, the slenderness governs the beam deformation under nonuniform bending. When variable, $H / L$ has a major impact on the pure shear stiffness which differs from that 
on the pure bending stiffness. As mentioned, the shear stiffness is a linear function of $H / L$, whereas in pure bending stiffness, the relation $k_{b}=f(H / L)$ is cubic. To express the dependence of the beam stiffness on the beam slenderness, we write $k_{s}$ and $k_{b}$ separately as:

$$
\begin{aligned}
& k_{s}=(5 \alpha / 6) \cdot G \cdot \psi_{S} \cdot(B(H / L)) \\
& k_{b}=(\beta / 12) \cdot E \cdot \psi_{I} \cdot\left(B(H / L)^{3}\right)
\end{aligned}
$$

By substituting the above relations in Eq. (1), we obtain the beam stiffness under non-uniform flexure:

$$
k=\frac{(5 \alpha \beta B / 6) \cdot E \cdot \psi_{I} \cdot G \cdot \psi_{S} \cdot(H / L)^{3}}{(10 \alpha) \cdot G \cdot \psi_{S}+(\beta) \cdot E \cdot \psi_{I} \cdot(H / L)^{2}}
$$

\section{The impact of slenderness}

Fig.1 illustrates how the beam resistance to shear and bending deformations, i.e. $\delta_{s}$ and $\delta_{b}$, changes with the beam slenderness. In the plot, curves of shear and bending deflection relative to the beam deflection, $\delta$, are shown for steel and iron beams of the rectangle family.

The curves confirm that for $0<H / L<0.1$, the stiffness of a prismatic beam is governed mainly by the bending strain. For relatively slender beams with $0.1<H / L<0.2$, the deflection due to shear increases by no more than $10 \%$ of the beam deflection. However, for deep beams, where $0.2<H / L<1$, the shear contribution is no longer negligible because it is greater than $10 \%$. For example, for a depth-to-span ratio of 0.5 , the shear contribution is approximately $40 \%$.

The horizontally scaled layers class exhibits a variation of the shear contribution with respect to $H / L$ equivalent to that of the solid rectangle. For the rectangles class of the proportionally scaled layers, the impact of slenderness depends on the size of the opening. The thinner the cross-section walls, the greater the shear deformation. Furthermore, as expected, the impact of slenderness for iron and steel is low, 
because the materials have similar properties. For example, stiffness contributions are equal at $H / L=0.566$ and $H / L=0.589$ respectively for prismatic steel and iron beams. On the other hand, a change of shape properties has a larger impact on the stiffness contributions. Hollow cross-sections undergo a significant shear deformation, which even for relatively slender beams cannot be systematically neglected.

\section{Performance Indices for minimum mass}

Based on the previous results, this section presents performance indices of minimum mass for pure shear stiffness design and non-uniform bending stiffness design.

\subsection{Pure Shear Stiffness}

In lightweight design, the minimum mass of a generic beam relative to that of a reference beam can be measured by a performance index, $p$. Maximizing $p$ results in minimizing mass.

Similar to the procedure followed for pure bending stiffness [9], $p$ is derived here for a pure shear stiffness requirement. The shape and material properties of the reference prismatic beam, as well as its mass, are assumed to be unity [9]. For prescribed shear stiffness, length, load and boundary conditions, the performance index for minimum mass of a generic beam, $p$, relative to the reference is given by:

$$
p=\frac{1}{m}=\frac{1}{u v \rho \cdot \psi_{A}}
$$

Where $u=B / B_{o}$ and $v=H / H_{o}$ are the envelope multipliers governing the scaling of a generic crosssection with $D(B, H)$ to the reference rectangle $D_{o}\left(B_{o}, H_{o}\right)$.

Since in pure shear stiffness design $k$ is specified, we write the shear stiffness ratio of a generic beam, $k$, to the reference beam, $k_{o}$, as 


$$
\frac{k}{k_{o}}=G \psi_{S} u v=1
$$

Rearranging Eq. (16) to substitute $u v$ in Eq. (15) gives the following performance index:

$$
p_{s}=\frac{G}{\rho} \cdot \frac{\psi_{S}}{\psi_{A}}
$$

Regardless of scaling, the performance in pure shear stiffness design is governed only by shape and material properties. For material selection, the shape is constant and $p$ reduces to $\frac{G}{\rho}$, whereas the performance for shape selection is given by:

$$
\lambda_{s}=\frac{\psi_{S}}{\psi_{A}}
$$

where $\lambda_{s}$ is the shear stiffness efficiency, analogous to that introduced for bending [13]. Fig. 2 illustrates in a bar chart the ranges of $\lambda_{s}$ for the shape families. The bounds are obtained by calculating the limit of $\lambda_{s}$ for $\psi_{A} \rightarrow 0$ and $\psi_{A} \rightarrow 1$ for each shape class. Although theoretical, because no manufacturing constraints and buckling requirements are considered, the ranges help select lightweight shapes in pure shear stiffness design. As expected, the best shape class in pure shear stiffness is no longer the most efficient in pure bending stiffness [13]. The vertically scaled shape class is indeed the least efficient for pure shear stiffness regardless of the shape family. On the other hand, the horizontally scaled layers classes are the most efficient in shear stiffness. Among the families of Table 1, the diamonds is the most efficient, followed by the ellipses.

\subsection{Non-uniform bending}


In non-uniform bending, the shear stiffness is coupled with the bending stiffness (Eq. (4)). A minimum mass criterion for the co-selection of material, shape, and slenderness of a beam can be obtained by combining Eq. (15) with (16). Hence, for a prescribed envelope, beam stiffness and boundary conditions, the performance $p$ of a beam is given by:

$$
p=\frac{1}{\rho \psi_{A}} \cdot \frac{\left(10 \alpha G+\beta E \cdot(H / L)^{2}\right) \cdot \psi_{I} \cdot \psi_{S}}{\left(10 \alpha G \psi_{S}+\beta E \psi_{I} \cdot(H / L)^{2}\right)}
$$

\section{Shape and Material Selection Charts}

Selection charts help to develop a visual understanding for the optimum design of structures. Maps are presented here to ease material, shape and slenderness co-selection in lightweight stiffness design.

\subsection{Pure Shear stiffness design}

Fig. 3 illustrates parametric plots of $G \psi_{S}$ versus $\rho \psi_{A}$ for beams in pure shear. For given envelope and increasing values, from 0 to 1 , of the parameters $c$ and $d$, the curves illustrate the resistance of alternative shapes to deform under shear strain. In contrast to pure bending, in pure shear the stiffness boundaries change. Not all shapes of the ellipse and diamond families fall within curves 1 and 2; rather only the rectangles classes are included in the domain. For a given material, higher shear stiffness can be obtained from the ellipse and diamond families because of a more efficient material distribution.

In pure shear stiffness, the upper and lower limiting curves of the rectangle family describe the horizontally and vertically scaled layers as opposed to those obtained in pure bending stiffness. For the rectangle class of horizontally scaled layers, the shear stiffness is linearly proportional to the amount of material, as it was for bending. However, for the proportionally scaled layers the performance drops and becomes the lowest for vertically scaled layers. This class is the least efficient because the cross-section core has no material to balance the maximum shear force. 
Similar behaviors are observed for ellipse and diamond shapes. However, the vertically scaled layers class for the ellipses and diamonds perform better than that of the rectangles. The reason is that the former shapes allow material distribution close to the neutral axis, where shear stress is maximum.

If the envelope is free to be scaled along prescribed directions, graphical selection in pure shear stiffness can be conveniently performed by using logarithmic charts. In this scenario, selection guidelines describing the performance for a given scaling condition are introduced in a way similar to bending. After taking the logarithmic function and rearranging, their equation from Eq. (17) can be written as:

$$
\log G \psi_{S}=\log \rho \psi_{A}+\log p
$$

In contrast to pure bending, in pure shear scaling does not impact the mass-efficiency. For any scaling condition, the iso-performance line has a slope equal to unity; the higher the y-intercept the better the performance. As an example, Fig. 4 shows three parallel lines corresponding to $p=0.25,0.5$ and 0.75 for shape selection. For a prescribed shear stiffness requirement, shapes above a line perform better than those below, regardless of the relative scaling of the envelopes.

\subsection{Non-uniform bending stiffness design}

For stiffness design of beams under non-uniform bending, pure shear stiffness is coupled with pure bending stiffness. With respect to slenderness the shear deformation scales with a law different than that for bending. For the former, deflection scales up linearly with H/L; whereas for the latter with the cube of H/L.

For a given envelope, Fig. 5, Fig. 6 and Fig. 7 illustrate the impact of varying slenderness on the stiffness of the shape families and their classes. Slenderness is assumed to be respectively: $H / L=10^{-4}$, $2 \cdot 10^{-1}, 5 \cdot 10^{-1}$ and, it is used with Eq. (19) to plot the performance for steel and iron, chosen as sample materials.

In Fig. 5, the curves plotted for slender beams match those obtained in previous works for pure bending $[9,10,13]$. Compared to this case, Fig. 6 shows that for a relatively slender beam, the performance 
of the most efficient shape in pure bending drops. For the class of the vertically scaled layers, the best shape in bending is the least efficient, since for this ideal shape the material does not fill the core, where the shear stress is highest. Fig. 7 plots the beam stiffness for beam with $H / L=0.5$. As can be observed, the curves are equivalent to those obtained for pure shear stiffness design (Fig. 3).

The charts presented in this work assist material and shape selection for lightweight stiffness of shortthick and slender beams. Through examination, insight can be gained into the impact of slenderness on beam stiffness and structural performance.

\section{Model Validation}

This section presents the numeric results obtained through Finite Element Analysis (software package: ANSYS () and compares them to those predicted by the model presented in this paper. The test case is that of a simply supported steel beam under a mid-span load $P=1000 N$. Cross-section dimensions are $B=0.1 \mathrm{~m}, H=0.2 \mathrm{~m}$, and material properties $\rho=7500 \mathrm{~kg} / \mathrm{m}^{3}, E=200 \mathrm{GPa}, G=75 \mathrm{GPa}$. The beams are modeled as beam elements (BEAM189) that can capture flexural, torsional, shear effects as well as warping effects. The numerical stiffness ratio $\left(k / k_{D}\right)_{\text {ANSYS }}$ for each tested beam is determined as:

$$
\left(k / k_{D}\right)_{A N S Y S}=\delta_{D \max _{-} A N S Y S} / \delta_{\max _{-} A N S Y S}
$$

with data $\delta_{D \max \_A N S Y S}$, for the envelope shape with $c=d=0$ and $\delta_{\text {max_ANSYS }}$, for the shape with prescribed $c$ and $d$.

Fig. 8 and Fig. 9 show FEA results obtained as Eq. (21) superimposed to the model plots. In Fig. 8, the vertically scaled rectangle concept, with $c=0.99$ and varying $d$ is plotted for varying depth-to-span ratios. On the other hand, Fig. 9 illustrates structural concepts for the proportionally and vertically scaled layers of the rectangle family. In both cases, a good agreement is observed between the model and numeric results. The errors vary in the percentage range of 0.01-6.28. The reason of the discrepancy is due to the beam element used in ANSYS®, which can also account for the warping effect. However, the divergence is 
within an acceptable range, especially for concept design. Similar results obtained for the families of the ellipses and diamonds confirm that the model is suitable for early optimization of beams under non-uniform flexure.

\section{Concluding Remarks}

This work extends a previous method for material and shape selection for the stiffness design of a beam under non-uniform bending. Cross-section size and shape, beam slenderness, and material govern the minimum mass of a beam, designed to meet a given stiffness requirement. The beam stiffness has been formulated as a combination of bending and shear stiffness. Shape transformers, introduced to model the pure shear correction factor, have been obtained by applying the strain energy method and Castigliano's theorem to alternative shape concepts.

The results have been used to develop design maps and efficiency bar charts that assist the co-selection of slenderness, shape and material for optimizing the lightweight design of stiff beams under non-uniform flexure. The results were validated by using finite element analysis to confirm that the model can be used appropriately at the concept stage of design, because the error falls within $6 \%$.

\section{Bibliography}

[1] Chow L, Conway HL., Winter G. Stresses in Deep Beams. Trans. Am. Soc. Civil Eng. 1952; 118: 686-708.

[2] Young WC, Budynas RG. Roark's Formulas for Stress and Strain. $7^{\text {th }}$ Ed. McGraw-Hill; 2002.

[3] Newlin JA, Trayer GW. The influence of the form of a wooden beam on its stiffness and strength-I:

Deflection of Beams with Special Reference to Shear Deformations. NACA Report 180; 1924.

[4] Timoshenko S, Young DH. Elements of Strength of Materials. $5^{\text {th }}$ Ed. D. Van Nostrand Company; 1968.

[5] Shanley FR .Weight-strength analysis of aircraft structures. $2^{\text {nd }}$ Ed. New York: Dover; 1960.

[6] Cox HL. Design of Structures of Least Weight. Pergamon Press; 1965. 
[7] Parkhouse JG. Structuring a process of material dilution. In: Nooshin H, editor. Proceedings of 3rd international conference on space structures. Elsevier Applied Science Publishers; 1984. p. 367-74.

[8] Ashby MF. Materials and Shape. Acta Metall. Mater 1991; 39 (6): 1025-1039.

[9] Pasini D, Smith DJ, Burgess SC. Selection of Arbitrarily Scaled Cross-Sections in Bending Stiffness Design. Proc Instn Mech Engrs Part L, J Mater: Design Appl; 2003; 217 (2): 113-25.

[10] Pasini D. Shape Transformers for Material and Shape Selection. Materials and Design 2007; 28 (7): 2071-2079.

[11] Ashby MF. Materials Selection in Mechanical Design. $3^{\text {rd }}$ Ed. Elsevier; 2005.

[12] Pasini D, Burgess SC, Smith DJ. Performance Indices for Arbitrarily Scaled Rectangular Crosssection in Bending Stiffness Design. Proc Instn Mech Engrs Part L, J Mater: Design Appl; 2002; 216 : 101 113.

[13] Pasini D. Shape and material Selection for Optimizing Flexural Vibrations in Multilayered Resonators. Journal of Microelectromechanical Systems, IEEE; 2006; 15(6): 1745-1758.

[14] Craig RR. Mechanics of Materials. $2^{\text {nd }}$ Ed. John Wiley \& Sons; 2000.

[15] Jenkins CHM, Khanna SK. Mechanics of Materials: A Modern Integration of Mechanics and Materials in Structural Design. Elsevier; 2005.

[16] Eskandari H. The effect of shear deformation on material selection for bending components. Materials and Design 2002; 24: 143-149.

[17] Boresi AP, Schmidt RJ. Advanced Mechanics of Materials. 6 ${ }^{\text {th }}$ Ed. John Wiley \& Sons; 2003.

[18] Renton JD. Generalized Beam Theory Applied to Shear Stiffness. Int. J. Solids Structures 1990; 27(15): 1955-1967.

[19] Timoshenko SP, Goodier J. Theory of Elasticity. McGraw-Hill; 1970.

[20] Wang CM, Reddy JN, Lee KH. Shear Deformable Beams and plates, Relationships with Classical Solutions. Elsevier; 2000.

[21] Hearn EJ. An Introduction to the Mechanics of Elastic and Plastic Deformation of Solids and Structural Materials. v. 1. $3^{\text {rd }}$ Ed. Butterworth-Heinemann; 1997.

[22] McGuire W, Gallagher RH, Ziemian RD. Matrix Structural Analysis. ${ }^{\text {nd }}$ Ed. John Wiley \& Sons; 2000 . 